\section{Inhibidor de las proteasas impide dispersión extraeritrocítica del parásito de la malaria}

Para reproducirse, los parásitos de la malaria copian su material genético después de penetrar en los eritrocitos y lo incorporan a merozoítos infectantes, que a su vez se acumulan en sacos protectores formados por las membranas de vacuolas parasitóforas (MVP), donde completan su ciclo vital asexual. Se sospecha, sobre la base de pruebas indirectas, que varias proteasas participan en el proceso de ruptura del eritrocito, durante el cual los merozoítos escapan al exterior de la célula huésped, desde donde invaden nuevos eritrocitos. Ahora, a raíz de un estudio en que se observó detenidamente el escape de los merozoítos hacia el espacio extracelular mediante inmunofluorescencia y microscopia electrónica, un grupo de investigadores ha llegado a la conclusión de que los inhibidores de las proteasas podrían servir de base para el tratamiento de la malaria, gracias a su capacidad para conseguir que las partículas infecciosas queden atrapadas dentro de un saco membranoso muy delgado fuera del eritrocito.

En el estudio los investigadores cultivaron eritrocitos infectados por esquizontes de Plasmodium falciparum en presencia de una cisteína (E64) que bloquea la actividad de ciertas proteasas. Después del tratamiento con E64, observaron que casi todos los parásitos se aglomeraban en sacos extraeritrocíticos recubiertos por membranas transparentes muy delgadas. Mediante la aplicación de anticuerpos fluorescentes a los cultivos, se encontró que estas membranas derivaban no del propio eritrocito, sino de la MVP. Se concluyó, por tanto, que el tratamiento con un inhibidor de las proteasas bloqueaba la salida de los parásitos al medio extraeritrocítico por detenimiento del proceso habitual de ruptura celular en una etapa intermedia. Una vez que este inhibidor se eliminaba del cultivo, los merozoítos recuperaban su capacidad para invadir nuevos eritrocitos y alcanzar su pleno desarrollo. (Salmon BL, Oksman A, Goldberg DE. Malaria parasite exit from host erythrocyte: A two-step process requiring extraerythrocytic proteolysis. Proc Natl Acad Sci USA 2001; 98(1):271-276.)

\section{La vacunación transcutánea: nuevo método de inmunización}

La inmunización transcutánea por medio de un parche con vacuna y coadyuvantes - aditivos que intensifican la respuesta inmunitaria, como la enterotoxina termolábil de Escherichia coli (LT) y la toxina de Vibrio cholerae (CT) - posee la ventaja de no ser una técnica invasora y de aplicarse a la piel, donde se produce una intensa actividad inmunitaria debido a la presencia de células de Langerhans en $25 \%$ de la superficie subepidérmica. En animales, esta técnica genera anticuerpos sistémicos y mucosos que confieren una fuerte protrección contra el antígeno de la vacuna.

La vacunación sin la necesidad de usar aguja hace falta con urgencia en el mundo, dado el alto riesgo de transmisión de enfermedades que se asocia con el uso y reuso de agujas y con su eliminación inadecuada. Otro mérito de la vacunación por parche cutáneo consiste en que la hidratación local producida por el parche disuelve fácilmente el estrato córneo y deja pasar el antígeno a la epidermis para ser conducido por las células de Langerhans hasta los nódulos linfáticos, donde se genera la respuesta inmunitaria de carácter sistémico.

Las sustancias TC y LT sirven como coadyuvantes y también como antígenos, ya que provocan la formación de anticuerpos contra sí mismas, lo cual confiere protección contra las enfermedades diarreicas. En un estudio reciente de fase I, se investigó la inmunogenia y la inocuidad de la $\mathrm{LT}$ administrada por vía transcutánea a 18 seres humanos. Cuatro voluntarios recibieron dosis de 25, 50 ó $250 \mu \mathrm{g}$ de LT; seis recibieron $500 \mu \mathrm{g}$. La LT se añadió a una almohadilla de gaza debajo de un parche adhesivo que se colocó sobre la piel del extremo proximal del brazo durante 6 horas. Uno, dos, tres y siete días después de la vacunación, cada voluntario fue observado para detectar cualquier reacción sistémica o local. A todos los voluntarios se les administró una dosis de refuerzo al cabo de 12 semanas. El grupo que recibió $500 \mu$ g de LT regresó para recibir una tercera dosis 35 semanas después de la primera, y $250 \mu \mathrm{g}$ de LT fueron administrados por parche a cada brazo.

No se detectaron reacciones adversas peligrosas. Solamente uno de los voluntarios tuvo una dermatitis local leve. En todos los sujetos que recibieron $500 \mu \mathrm{g}$ se cuadruplicaron los anticuerpos séricos de tipo IgG contra LT y se produjeron respuestas intensas a las dosis de refuerzo. La IgG se mostró levemente aumentada en las personas que recibieron otras dosis de vacuna. Los anticuerpos contra LT fueron persistentes y pudieron detectarse hasta la trigesimoquinta semana en el grupo que re- 
cibió $500 \mu \mathrm{g}$. Después de la tercera inmunización, se obtuvo un aumento de 14,6 veces en los anticuerpos IgG contra LT y de 7,2 veces en los anticuerpos IgA. Estos resultados indican que la técnica de vacunación transcutánea provoca una respuesta inmunitaria intensa y persistente y se presta para uso generalizado. Se trata de una técnica sencilla y práctica que puede mejorar la seguridad y la eficacia de la vacunación. (Glenn GM, Taylor DN, Li Xiuru, Frankel S, Montemarano A, Alving CR. Transcutaneous immunization: A human vaccine delivery strategy using a patch. Nature Med 2000; 6(12):1403-1406.)

\section{La glucohemoglobina $\left(\mathrm{HbA}_{1 \mathrm{c}}\right)$ en la predicción del riesgo de trastornos cardiovasculares}

En un estudio prospectivo de base poblacional efectuado en Cambridge, Inglaterra, un grupo de investigadores descubrió que la concentración de glucohemoglobina $\left(\mathrm{HbA}_{1 \mathrm{c}}\right)$, marcadora de la concentración de glucosa en la sangre, tiene un valor predictivo con respecto a trastornos cardiovasculares similar al de la tensión arterial y la colesterolemia. Los sujetos estudiados fueron 4662 hombres entre las edades de 45 y 79 años que habían acudido a un examen físico y a medirse la glucemia como parte de la Investigación Prospectiva Europea del Cáncer y la Nutrición en Norfolk, Inglaterra. Los hombres fueron divididos en cinco grupos: el primero se compuso de aquellos con diabetes ya conocida y confirmada; el segundo, de hombres con diabetes confirmada durante el estudio, pero desconocida previamente; los otros tres grupos fueron integrados por el resto de la población estudiada, dividida en tres partes determinadas por sus concentraciones de $\mathrm{HbA}_{1 c^{\prime}}$ atendiendo a puntos de corte clínicamente importantes.

Se encontró que los hombres diabéticos tenían un mayor riesgo de morir de cualquier causa, de trastornos cardiovasculares y de enfermedad coronaria que los hombres que no tenían diabetes. Tanto en los diabéticos como en los hombres sanos, el riesgo de morir aumentaba en la medida en que aumentaba la $\mathrm{HbA}_{1 \mathrm{c}}$.

Tales resultados tienen grandes implicaciones desde el punto de vista de la salud pública, ya que una reducción a nivel poblacional de apenas $0,1 \%$ ó $0,2 \%$ de la concentración de glucosa en la sangre puede reducir la mortalidad total en 5\% y $10 \%$, respectivamente. Las medidas preventivas deben encamínarse a reducir la concentración promedio de glucosa en la sangre de toda la población mediante un estilo de vida sano, una buena alimentación y abundante ejercicio. (Khaw KT, Wareham N, Luben R, Bingham S, Oakes S, Welch, et al. Glycated hae- moglobin, diabetes, and mortality in men in Norfolk cohort of European Prospective Investigation of Cancer and Nutrition (EPIC-Norfolk). Br Med J 2001;322:15.)

\section{Vacuna contra el cólera muestra eficacia en animales}

Cualquier vacuna contra el cólera, si ha de ser eficaz, debe elaborarse a partir de vibriones vivos, pero se corre el riesgo de que Vibrio cholerae prolifere en el medio ambiente, con posibles consecuencias peligrosas para los seres humanos. Son promisorias en este sentido las recientes investigaciones efectuadas en voluntarios cubanos por Rafael Fando y sus colaboradores, del Centro Nacional de Investigaciones Científicas en La Habana, quienes crearon una forma mutante de $V$. cholerae $638 \mathrm{El}$ Tor Ogawa incapaz de proliferar en medios sin timidina debido a la ausencia de un gen funcional (thyA) que codifica la elaboración de la sintetasa del timidilato. Esta variante de $V$. cholerae, a la cual le pusieron el nombre de 638T, no pudo sobrevivir en aguas sin timidina y al cabo de 11 días de incubación en aguas negras sus concentraciones se habían reducido a niveles indetectables. En cambio, una variante de 638T provista de un plásmido aportador de timidina ( $p V T 1)$ sí sobrevivió en aguas residuales por espacio de 18 días. La capacidad para sobrevivir en medios desprovistos de timidina es el único rasgo que distingue a 638 de 638T; ambas variantes son fenotíopicamente iguales en su ritmo de proliferación, motilidad, capacidad para colonizar el organismo de ratones recién nacidos y capacidad inmunógena en conejos adultos.

Estos resultados apuntan hacia la factibilidad de elaborar una vacuna contra el cólera a partir de vibriones vivos atenuados pero desprovistos de la capacidad para proliferar en el medio ambiente, con lo cual se elimina el peligro de transmisión que hasta ahora ha preocupado a la comunidad científica. (Valle E, Ledon T, Cedre B, Campos J, Valmaseda T, Rodríguez B, et al. Construction and characterization of a nonproliferative El Tor cholera vaccine candidate derived from strain 638. Infect Immunol 2000;68(11):6411-6418.)

\section{Efectos a largo plazo de la reducción de peso sobre la tensión arterial}

Aunque el tratamiento farmacológico de la hipertensión reduce significativamente la morbilidad y mortalidad relacionadas con las enfermedades cardiovasculares, su administración a largo plazo 
puede tener efectos indeseables y requiere vigilancia médica continua, por lo que las intervenciones para modificar el estilo de vida siguen constituyendo una estrategia fundamental en la prevención primaria y en el tratamiento inicial de la hipertensión. La reducción del peso corporal parece ser un método eficaz de prevención primaria, pero sus efectos a largo plazo sobre la tensión arterial todavía no han sido estudiados de forma exhaustiva.

En este informe se describen los resultados obtenidos con la reducción de peso en los Ensayos Clínicos sobre la Prevención de la Hipertensión, fase II (TOHP II), una serie de ensayos controlados aleatorizados en los que se investigaron los efectos antihipertensores de la reducción del peso corporal y del sodio en la dieta, individualmente o en combinación, a lo largo de un período de 3 a 4 años. El informe se centra únicamente en dos de los cuatro grupos: el asignado al programa de reducción de peso $(n=595)$ y el grupo testigo $(n=596)$.

Los participantes fueron personas de ambos sexos, de 30 a 54 años de edad, con tensión arterial diastólica de 83 a $89 \mathrm{~mm} \mathrm{Hg}$ y sistólica de menos de $140 \mathrm{~mm} \mathrm{Hg}$, que al inicio del estudio tenían un peso 10 a $65 \%$ superior al ideal. Se excluyeron las embarazadas y los individuos tratados con medicamentos que pudieran influir sobre la tensión arterial o con signos clínicos o de laboratorio de enfermedad cardiovascular, diabetes sacarina o insuficiencia renal.

La intervención consistió en un programa de 3 años de asesoramiento individual y grupal para modificar los hábitos dietéticos y aumentar la actividad física, y de apoyo social para mantener los cambios. El objetivo consistió en conseguir una reducción de peso de 4,5 $\mathrm{kg}$ durante los primeros 6 meses y mantenerla durante el resto del estudio.

El peso corporal y la tensión arterial fueron registrados semestralmente por personal que desconocía el grupo al que había sido asignado cada individuo. Estas determinaciones se repitieron a lo largo de 36, 42, o 48 meses, dependiendo de la fecha de entrada en el estudio. Se analizaron las diferencias entre los grupos a los 6, a los 18 y a los 36 meses. Las tasas de seguimiento a los 36 meses fueron elevadas: $92 \%$ en el grupo de estudio y $93 \%$ en el grupo testigo para las mediciones de peso, y 89 y $86 \%$, respectivamente, para las mediciones de la tensión arterial.

Las modificaciones del peso fueron significativamente diferentes en los grupos, tanto a los 6 como a los 18 y a los 36 meses: en el grupo de estudio el peso disminuyó en 4,4, 2,0 y 0,2 kg, respectivamente, mientras que en el grupo testigo aumentó en $0,1,0,7$ y $1,8 \mathrm{~kg}$.

La tensión arterial, tanto diastólica como sistólica, fue significativamente menor en el grupo de estudio que en el grupo testigo a los 6, a los 18 y a los 36 meses. El riesgo relativo de hipertensión en el grupo de estudio fue de 0,58 (intervalo de confianza de $95 \%$ [IC95\%]: 0,36 a 0,94) a los 6 meses, 0,78 (IC95\%: 0,62 a 1,00) a los 18 meses, y 0,81 (IC95\%: $0,70$ a 0,95$)$ a los 36 meses. Como era de esperar, las mayores reducciones de la tensión arterial correspondieron a los individuos que perdieron más peso $\mathrm{y}$, en particular, a los que consiguieron una reducción de al menos $4,5 \mathrm{~kg}$ a los 6 meses y la mantuvieron durante los 30 meses siguientes. En estos individuos el riesgo relativo de hipertensión fue de 0,35 (IC95\%: 0,20 a 0,59), frente a 0,75 (IC95\%: 0,53 a 1,04) en los que perdieron al menos $4,5 \mathrm{~kg}$ a los 6 meses, pero que a los 36 meses habían perdido menos de $2,5 \mathrm{~kg}$, y 1,10 (IC95\%: 0,85 a 1,42) en los que habían perdido $2,5 \mathrm{~kg}$ o menos a los $6 \mathrm{y}$ a los 36 meses.

Estos resultados, coincidentes con los de estudios anteriores, la mayoría de ellos con menores períodos de observación, indican que la disminución del peso corporal, aunque sea moderada, permite obtener reducciones clínicamente significativas y a largo plazo tanto de la tensión arterial como del riesgo de hipertensión. No obstante, todavía no se ha resuelto el problema del escaso éxito de estos programas en lo que se refiere al mantenimiento de la pérdida de peso a largo plazo. Así en este estudio, solo $13 \%$ de las personas fueron capaces de mantener una reducción de peso de 4,5 kg o más al cabo de 3 años. Además, hay que tener en cuenta que, a pesar de que en estas personas la reducción de la tensión diastólica fue sostenida con una reducción sostenida del peso, no ocurrió lo mismo con la tensión sistólica, que volvió a aumentar con el tiempo a la misma velocidad que en el grupo testigo. Esto indica que la pérdida de peso, aunque sea sostenida, puede no ser suficiente para prevenir el aumento de la tensión sistólica relacionado con la edad, y que parecen existir otros determinantes importantes de la tensión arterial, como la ingesta de sodio.

De acuerdo con los resultados de este estudio, se debería seguir fomentando la pérdida de peso como medida para prevenir la hipertensión, aunque es necesario desarrollar nuevos programas de reducción del peso con mejores resultados a largo plazo. (Stevens VJ, Obarzanek E, Cook NR, Lee I-M, Appel LJ, Smith West D, et al. Long-term weight loss and changes in blood pressure: results of the Trials of Hypertension Prevention, Phase II. Ann Intern Med 2001;134:1-11)

\section{Controversia sobre el programa de vacunación antigripal en masa en la provincia canadiense de Ontario}

En dos artículos publicados recientemente en la sección "Controversias" del Canadian Medical As- 
sociation Journal se presentan dos puntos de vista contrapuestos acerca del programa de vacunación en masa contra la gripe que ha decidido poner en marcha el gobierno de la provincia canadiense de Ontario.

Uno de los autores, consultor temporal de un fabricante de vacunas antigripales, favorece la implantación del programa, que en su opinión constituye una ampliación sensata y lógica de los programas ya existentes de vacunación de las poblaciones en alto riesgo. Sus argumentos son, básicamente, los siguientes:

La gripe es una enfermedad infecciosa de gran importancia para la salud pública, que afecta cada año a 10 a $20 \%$ de la población adulta y hasta a $40 \%$ de la población pediátrica, y que causa miles de muertes. Las pandemias de gripe constituyen las emergencias de salud pública predecibles más importantes y la vacunación es actualmente la única forma eficaz de combatirlas.

La vacuna antigripal es segura, eficaz y muestra una buena relación entre costes y beneficios. Debido a las variaciones antigénicas del virus de la gripe, la vacuna tiene que ser modificada periódicamente, de acuerdo con las previsiones de cuál será la cepa predominante cada año, lo cual implica la necesidad de revacunación anual, pero, a pesar de este inconveniente, tiene una eficacia de 70 a $90 \%$ en adultos sanos y los efectos colaterales graves son muy raros.

Hay dos razones por las cuales la actual estrategia de vacunación de las poblaciones en alto riesgo es ineficaz para proteger a los individuos con alto riego de complicaciones: las bajas tasas de cobertura vacunal, especialmente en personas jóvenes con problemas médicos crónicos, y la ineficacia de esta estrategia para limitar la expansión de la gripe y su ineficacia frente a las pandemias.

Aunque la vacunación es beneficiosa desde el punto de vista individual, desde el punto de vista poblacional sus beneficios dependen del número de individuos inmunizados. A pesar de que es improbable que las actuales vacunas antigripales consigan bloquear completamente la transmisión de la gripe, como sucedió con la poliomielitis, cuanto más individuos haya inmunizados menos serán los susceptibles, capaces de exponer a otros a la infección. En este aspecto, la vacunación de los escolares sería particularmente importante, dada la facilidad con la que transmiten la enfermedad a otros. De hecho no hay nada nuevo en esta estrategia, cuya lógica es la misma que la de la recomendación de vacunar a las personas que, como el personal sanitario, pueden transmitir la gripe a los individuos en alto riesgo.
Como nunca se ha intentado la vacunación a esta escala, el autor reconoce que todavía hay muchas incertidumbres en estos argumentos y que este programa del estado de Ontario debería ser cuidadosamente evaluado. No obstante, en el mejor de los casos esta estrategia constituiría un importante avance en el control de la gripe; en el peor, no sería eficiente y tendría que modificarse.

Para el otro autor, antes de adoptar una medida de este tipo es necesario disponer de datos que fundamenten la decisión, cosa que, en su opinión, no ocurre.

En primer lugar destaca que todavía no se sabe qué proporción de casos de "síndrome gripal" son realmente debidos a los virus de la gripe A y B, aunque los escasos estudios existentes indican que probablemente sea inferior a $10 \%$, lo cual limita enormemente el valor de los estudios sobre el impacto poblacional de la vacuna.

Con respecto a la eficacia, una revisión sistemática de 20 ensayos clínicos ha indicado que las vacunas parenterales inactivadas tienen una eficacia de $68 \%$ (intervalo de confianza de $95 \%$ (IC95\%): 49 a $79 \%$ ), medida por su capacidad para inducir anticuerpos eficaces contra el virus, pero solo de 24\% (IC95\%: 14 a 33\%) para reducir el número de casos clínicos de "síndrome gripal", con lo cual solo uno de cada cuatro individuos vacunados adquiere protección frente a la enfermedad clínica. Aunque la vacunación reduce significativamente el absentismo laboral, la magnitud del efecto ( 0,4 días) es clínicamente irrelevante. Los pocos ensayos clínicos que analizaron la tasa de complicaciones no mostraron una reducción significativa. Estos datos discrepan en parte de los obtenidos en un metaanálisis de los efectos de la vacunación en ancianos, que mostró mayor eficacia clínica, apoyando así la estrategia actual de vacunar únicamente a los ancianos y a otros grupos en alto riesgo.

Desde el punto de vista de la eficiencia, los datos existentes son contradictorios. En la presentación del programa de vacunación en Ontario se cita un estudio de costes y beneficios según el cual el beneficio sería de aproximadamente US $\$ 40$ por vacunación, mientras que otro estudio realizado en soldados británicos concluye que el coste de la prevención de un caso de gripe cuesta aproximadamente US\$ 6200.

Teniendo en cuenta la dinámica de las epidemias de gripe, es improbable que el programa de Ontario proporcione un control sobre la extensión de la gripe en la comunidad. Para lograr este objetivo sería necesario vacunar a más de $90 \%$ de la población, meta que es muy improbable que se alcance. 
Considerando la calidad de la información existente y el coste de la vacunación universal, este autor cree que la decisión del gobierno de Ontario probablemente no sea seguida por otros gobiernos. Por último, duda que alguna vez se llegue a conocer el efecto real de este programa y su relación coste-eficacia, puesto que no ha sido diseñado de forma que permita una evaluación rigurosa. (Schabas RE. Mass influenza vaccination in Ontario: a sensible move. CMAJ 2001;164:36-37; Demicheli V. Mass influenza vaccination in Ontario: Is it worthwhile? CMAJ 2000;164:38-39).

\section{Efecto de una dieta baja en sal en la tensión arterial}

Es un hecho ampliamente conocido que el consumo de sal tiene un efecto directo en la tensión arterial, pero según revelan los resultados de un estudio reciente efectuado por Sacks y colaboradores de la Universidad de Harvard, el efecto es mucho mayor de lo imaginado hasta el momento. Los investigadores determinaron el resultado, en un total de 412 personas hipertensas y normotensas, de consumir diferentes cantidades de sodio en la dieta, en combinación con el régimen dietético DASH (Dietary Approaches to Stop Hypertension), que es rico en verduras, frutas y productos lácteos con bajo contenido de grasa. Se asignaron aleatoriamente dos tipos de alimentación: la dieta típica estadounidense al grupo testigo, y el régimen DASH al grupo de estudio. Dentro de cada uno de estos dos grupos, la dieta se acompañó de un aporte de sal elevado, moderado o bajo por espacio de 30 días consecutivos, siguiendo un patrón aleatorio.
El paso de un consumo de sal elevado a un consumo intermedio se asoció con una reducción de la tensión arterial sistólica de $2,1 \mathrm{mmHg}(P<$ 0,001 ) en el grupo testigo (dieta habitual) y de 1,3 $\mathrm{mmHg}$ en el grupo de estudio (régimen DASH) $(P=0,03)$. Al reducirse el consumo de sodio de un nivel moderado a un nivel bajo, se produjo una reducción adicional de la tensión arterial de 4,6 $\mathrm{mmHg}$ en el grupo testigo $(P<0,001)$ y de 1,7 $\mathrm{mmHg}$ en el grupo de estudio $(P<0,01)$. Estos efectos se observaron en personas normotensas e hipertensas de todas las razas y de ambos sexos. Como puede observarse, al reducir la cantidad de sodio, diferencias significativas se produjeron en las personas sometidas al régimen DASH, y estas diferencias fueron más marcadas en las que consumían mayores cantidades de sodio. Cuando se comparan las personas en extremos opuestos -es decir, las que ingirieron una dieta habitual rica en sodio y las que se sometieron a la dieta DASH con bajo consumo de sodio-, se observa una diferencia de tensión arterial mayor de 7,1 mmHg en normotensos y de 11,5 $\mathrm{mmHg}$ en hipertensos.

Los autores concluyen que tanto el régimen DASH como un consumo de sodio en la dieta de menos de $100 \mathrm{mmol}$ al día reduce la tensión arterial notablemente, pero el efecto es mayor cuando ambas conductas se combinan. Los beneficios a largo plazo dependerán de la capacidad de las personas para cambiar sus hábitos alimentarios permanentemente y de la disponibilidad de alimentos con un bajo contenido de sodio. (Sacks FM, Svetkey LP, Vollmer WM, Appel LJ, Bray GA, Harsha D, et al. Effects on blood pressure of reduced dietary sodium and the Dietary Approaches to Stop Hypertension (DASH) diet. New Engl J Med 2001;344(1): 3-10.) 